\title{
Deliberación democrática y gobernanza en la participación ciudadana local. El caso de los Consejos Comunales de la Sociedad Civil (cosoc), Biobío, Chile
}

\author{
JaVier ANTONio LeÓn AravenA* \\ ESAU AARON Figueroa SILVA**
}

Artículo recibido: $1^{\circ}$ de febrero de 2019

Artículo aprobado: 5 de junio de 2019

Doi: https://doi.org/10.12804/revistas.urosario.edu.co/desafios/a.7219

Para citar este artículo: León Aravena, L. A., \& Figueroa Silva, E. A. (2020). Deliberación democrática y gobernanza en la participación ciudadana local. El caso de los Consejos Comunales de la Sociedad Civil (COSOC), Biobío, Chile. Desafios, 32(2), 1-38. https://doi. org/10.12804/revistas.urosario.edu.co/desafios/a.7219

\section{Resumen}

El establecimiento de espacios de participación es un desafio abierto para gran parte de los Estados latinoamericanos. Su óptimo funcionamiento dependería de factores adicionales a la gestión institucional. Este artículo indaga cómo los Consejos Comunales de Organizaciones de la Sociedad Civil (COSOC) responden a requerimientos de gobernanzay deliberación democrática, que articulados con elementos de capital social y cultural, son componentes fundamentales del ejercicio de participación ciudadana.

\footnotetext{
* Universidad del Bío-Bío (Concepción, Chile). Correo electrónico: jleon@ubiobio.cl. ORCID: http://orcid.org/0000-0003-4491-689X

** Universidad del Bío-Bío (Concepción, Chile). Correo electrónico: efigueroa@ubiobio. cl. ORCID: http://orcid.org/0000-0002-1040-0433
} 


\title{
2 I Javier Antonio León Aravena - Esau Aaron Figueroa Silva
}

Para explorar este planteamiento, se tomó el caso del Consejo de la Sociedad Civil (COSOC), Chile. Los datos surgen del proyecto para la instalación del COSOC (Ley 20 500), ejecutado en la región del Biobio, Chile, en 2017. La metodología es mixta, predominantemente cualitativa, basada en el análisis de talleres participativos con apoyo de datos descriptivos tomados de las encuestas. Los principales resultados apuntan a la operatividad y eficacia de la participación y al valor que esta adquiere a partir de las condiciones del contexto y demanda social, es decir, cuestiones como la información disponible, el nivel de asociatividad, la formación cívica, las asimetrías de poder, entre otras. Las anteriores, variables vinculadas a la estructura y condiciones de los espacios promovidos y a la calidad de los procesos democráticos en la región.

Palabras clave: democracia deliberativa; gobernanza; participación ciudadana; política local; sociedad civil; Chile.

\section{Democratic Deliberation and Governance in Local Citizen Participation. The Case of the Communal Councils of Civil Society (cosoc), Bíobío, Chile}

\begin{abstract}
Citizen participation is an open challenge for a large part of the Latin American states. Its optimal functioning would depend on various factors in addition to institutional management. This article seeks to investigate how the Communal Councils of Civil Society Organizations (COSOC) respond to the requirements of democratic governance and deliberation, proposed as fundamental components for the proper exercise of citizen participation articulated with elements of social and cultural capital. To investigate this approach, we took the case of Chile and the local experience of COSOC (Law 20 500), using data from the project "Installation of Civil Society Councils and Proposal of Construction of two mechanisms of Citizen Participation according to reality territorial", executed in the Biobio Region, Chile, in 2017. The methodology is mixed, predominantly qualitative, based on the analysis of participatory workshops and the support of descriptive data from surveys. The main results point to the operation and effectiveness of the participation and the value that it acquires based on the conditions of the context and social demand. That is to say, issues such as the information available, the level of associativity, civic
\end{abstract}


training, power asymmetries, etc. All of them are variables linked to the structure and conditions of the promoted spaces and the quality of their democratic processes. Keywords: Democratic deliberation; governance; citizen participation; civil society councils; Chile.

\section{Deliberação democrática e governança na participação cidadã local. O caso dos Conselhos Comunais da Sociedade Civil (cosoc), Biobío, Chile}

\section{Resumo}

Aparticipação é um desafio aberto para grande parte dos Estados Latino-Americanos. Seu ótimo funcionamento dependeria de fatores adicionais à gestão institucional. Este artigo indaga como os Conselhos Comunais de Organizaçoes da Sociedade Civil (COSOC), respondem a requerimentos de governança e deliberação democrática, que articulados com elementos de capital social e cultural, são componentes fundamentais do exercício de participação cidadã. Para explorar esta abordagem, tomou-se o caso do Conselho da Sociedade Civil (COSOC), Chile. Os dados surgem do projeto para a instalação do COSOC (Lei 20 500), executado na Região do Biobio, Chile, em 2017. A metodologia é mista, predominantemente qualitativa, baseada na análise de workshops participativos, com apoio de dados descritivos de inquéritos. Os principais resultados apontam à operatividade e eficácia da participação e o valor que esta adquire a partir das condições do contexto e demanda social. Isto é, questões como a informação disponivel, o nível de associatividade, a formação cívica, as assimetrias de poder, etc. variáveis vinculadas à estrutura e condições dos espaços promovidos e a qualidade dos processos democráticos.

Palavras-chave: democracia deliberativa; governança; participação cidadã; política local; sociedade civil; Chile.

\section{Introducción}

Diversos estudios señalan la importancia y beneficios de la participación ciudadana. De hecho, las actuales tendencias asociadas a la gestión pública y político-estatal apuntan a su robustecimiento en 


\section{I Javier Antonio león Aravena - Esau Aaron figueroa Silva}

todo el ciclo de las políticas públicas (Aguilar, 2007; Blanco, Fleury \& Subirats, 2012; Sánchez, 2015). En función de ello se ha intentado reestructurar las lógicas institucionales de la gestión pública, pero también las relaciones entre Estado y sociedad civil (Dagnino, Olvera \& Panfichi, 2006).

En términos básicos, lo anterior constituye un desafío para la mayoría de los países latinoamericanos (Chamorro, 2016; Cruz \& Mballa, 2017) porque, al tiempo que se consolidaron los procesos de refundación democrática, las posdictaduras (Levine \& Molina, 2007), también lo hizo un neoconservadurismo institucional, el cual, sustentado en un liberalismo representativo, propició un distanciamiento entre lo social y político (Escobar, 2004). Es decir, en tanto se ampliaron derechos políticos, el ejercicio de derechos económicos y sociales se dificultó para amplios sectores de la población. Como consecuencia, ha crecido la desconfianza hacia las nuevas democracias (Chaguaceda \& Caldera, 2016), fenómeno que ha sido calificado por O’Donnell (2004) como ciudadania de baja intensidad. Lo anterior pone en riesgo el papel protagónico que la participación ciudadana debería cumplir en la consolidación del Estado democrático (Cunill, 1991).

Si bien se han tomado acciones para subsanar esta situación, lo cierto es que la sola oferta institucional del Estado no parece efectiva si no se consideran antes las condiciones de demanda social (Canto, 2008). Se requiere, a su vez, de una revisión de las lógicas y procedimientos a través de los cuales se configura el ejercicio participativo. En función de ello se destacan dos conceptos: primero, el de gobernanza, es decir, el proceso otorgador de legitimidad en el que diversos actores, de manera conjunta, normada, e inmersos en una modo de regulación heterárquico, toman decisiones referidas a cuestiones que involucran intereses particulares y colectivos (Le Galès, 2009; Zurbriggen, 2011). Segundo, el de democracia deliberativa, concebida como un contexto de expansión de la esfera pública y un modelo en el que la toma de decisiones está determinada por el desarrollo de diálogos fundados en la igualdad de los sujetos involucrados (Brugué, 2009; León, 2014). 
$\mathrm{Al}$ respecto, Chile se presenta como un caso interesante, ya que si bien ha sido catalogado como el segundo país más democrático de la región ${ }^{1}$ (The Economist, 2017), también es un lugar de distanciamiento entre mundo político y sociedad civil, situación que ha desmotivado la participación ciudadana (De la Maza, 2011b). Esto resulta comprensible si se considera la historia reciente de la sociedad civil, que ha transitado desde la coerción de la dictadura militar (1973-1989) a la subvaloración durante la transición democrática. Adicionalmente, se figura un diseño democrático en el que la ciudadanía es vista como receptora de beneficios y derechos, más no como un actor decisivo (De la Maza, Robles, Montecinos \& Ochsenius, 2012), generándose así apatía y desconfianza social (Herrmann \& Klaveren, 2016).

En este contexto se promulgó en 2011 la Ley 20 500, Sobre Asociaciones y Participación Ciudadana en la Gestión Pública, la cual regula y promueve la participación ciudadana en diversos ámbitos. Este cuerpo legal creó un nuevo órgano a nivel local, denominado Consejo Comunal de Organizaciones de la Sociedad Civil (COSOC) que, si bien pareció responder a los requerimientos necesarios para consolidar la participación ciudadana, particularmente desde la óptica de una gobernanza institucionalizada, ha presentado problemas que reflotan los cuestionamientos sobre la correcta articulación entre condiciones de oferta y demanda participativa, pero, sobre todo, cuestionamientos en torno a su constitución y funcionamiento. Con esta Ley también se creó un Consejo para los Servicios de otras Reparticiones del Estado (regionales y nacionales también denominado COSOC, Consejo de la Sociedad Civil); sin embargo, estos no serán objeto de este análisis.

Así, la implementación del cosoc, del nivel comunal, aparece como un fenómeno interesante para analizar, principalmente, por el papel conferido a los mecanismos de participación local, concebidos como una medida estratégica para enfrentar la crisis de representación que afecta a la mayoría de las democracias latinoamericanas (Pressacco \& Rivera, 2015).

1 Superado solo por Uruguay. Chile ocupó el puesto 26 y Uruguay el puesto 17. 


\section{I Javier Antonio León Aravena - Esau Aaron figueroa Silva}

Entonces, ¿cómo responde el COSOC a un ejercicio de gobernanza que reúna las condiciones para generar procesos efectivos de deliberación democrática, y por tanto, de óptima participación ciudadana? Para responder a esta pregunta, en este artículo de investigación, nos centraremos en dar cuenta del caso de estudio del proyecto titulado "Instalación de Consejos de la Sociedad Civil y Propuesta de Construcción de dos mecanismos de Participación Ciudadana de acuerdo a la realidad territorial", ejecutado por la Universidad del Bío-Bío, en la región del Biobío, Chile, durante el año 2017.

En este se conceptualiza la participación ciudadana en Chile, para luego indagar específicamente por las condiciones de implementación del COSOC en la región mencionada. A continuación, presentaremos el contexto, posteriormente el cuerpo teórico que fundamenta el análisis, seguido del cual se detalla el caso de estudio, la metodología utilizada y la exposición de los principales datos y resultados. Finalmente, las conclusiones exponen los hallazgos más significativos.

\section{Participación ciudadana y Estado en Chile}

En un sentido clásico, la participación puede ser concebida como la forma a través de la cual los individuos intervienen en actividades, generalmente vinculadas a decisiones del quehacer público, representando los intereses de la sociedad de la que hacen parte (Cunill, 1991). Se debe recordar que la participación es un concepto sujeto a diversas manifestaciones e interpretaciones, y que puede presentarse en formatos pasivos, restringidos, deliberativos, institucionales, entre otros (Welp, 2016). La forma que ella adquiera estará determinada por la existencia, o no, de mecanismos institucionalizados que la prescriban (Garrido-Vergara, Valderrama \& Peñafiel, 2016).

En Chile, la necesidad de promover la participación en la gestión pública se viene replanteando con fuerza desde fines del siglo XX. Esto ha suscitado una serie de iniciativas políticas, que, no obstante, se han visto limitadas en sus resultados (Loyola, 2016). La existencia de una ciudadanía más informada y exigente requiere de mejores 
mecanismos y condiciones que favorezcan la participación. Esto considerando, además, la trayectoria histórica de la sociedad civil en Chile, la cual no ha contribuido a fortalecer sustantivamente los lazos con el Estado. Al respecto, se debe considerar la instrumentalización de la sociedad chilena y el rol institucional pasivo que se le ha atribuido en diferentes gobiernos (De la Maza et al., 2012).

Esta preocupación ha sido tomada en cuenta por el Estado chileno, el cual es más consciente de los cambios culturales y políticos por los que atraviesa la sociedad, y, por tanto, de los nuevos desafíos asociados a su régimen de gobierno. Lo anterior ha requerido proponer nuevas medidas que fortalezcan y den soporte institucional, con el fin de garantizar que la ciudadanía tenga acceso a las decisiones públicas. Así pues, se ha tenido la intención de hacer diversos cambios, no solo en materia de participación ciudadana, sino también, en asuntos de transparencia y acceso a la información. Sin embargo, esta nueva tendencia, caracterizada por su mayor flexibilidad y por la implementación de más instancias de diálogo, se ha topado con una tradición de gobierno centralista y verticalizada, en la que las políticas públicas son concebidas y producidas desde el esquema "arriba-abajo" (topdown), con procesos ciudadanos acotados, o más bien reactivos, y con baja permeabilidad democrática (Salazar, 2005).

Los desafíos para concretar formas de participación efectivas en Chile apuntan al perfeccionamiento de indicadores que den cuenta de las instancias de comunicación entre Estado y ciudadanía, así como también a una profundización en la morfología (necesidades, calidad, equidad, acceso, estructura, entre otras) misma de la participación, de modo que esta no se transforme en un mero requerimiento normativo derivado de la oferta estatal. Para ello se requiere no solo promover la institucionalización o integrar la participación al ciclo de las políticas públicas, sino también salvaguardar situaciones de exclusión que se puedan dar en el espacio mismo de la participación, cuestión que traslada la discusión al ámbito de la calidad de los procesos y a su acceso democrático (De la Maza, 2008), y, por tanto, a los postulados de la gobernanza y la deliberación democrática. 


\section{Institucionalidad y marco normativo de la participación ciudadana en Chile}

La normativa en Chile es diversa e incluye referencias a la incorporación de la participación en la gestión. Así pues, vale la pena destacar la Ley 18575 Orgánica de Constitucional de Bases de la Administración del Estado, la cual consagra el derecho a la participación ciudadana en la gestión pública. También está la Ley 19175, Orgánica de Constitucional sobre Gobierno y Administración Regional que define un órgano regional de representación ciudadana a través del Consejo Regional. Por último, durante el año 2011, se promulgó la Ley 20500 sobre Asociaciones y Participación Ciudadana en la Gestión Pública. En ella se sistematizaron diversos instrumentos y experiencias desarrolladas a nivel sectorial y territorial y se estableció un marco normativo relacionado con el acceso a la información y con la asociatividad de los ciudadanos. En este se reconocía, específicamente, el derecho de las personas a participar en las políticas, planes, programas y acciones ejecutadas.

En los años recientes, se ha continuado con la realización de acciones para fortalecer esta dimensión, entre los cuales se destaca el Instructivo Presidencial 007 para la participación ciudadana en la gestión pública, del año 2014. Ello implicó revisar y perfeccionar los mecanismos existentes; integrar de forma transversal el enfoque de participación a todos los órganos de las Administración Pública; fortalecer la institucionalidad ciudadana; $y$, finalmente, promover la participación con un enfoque de derecho, al respetar la diversidad social y cultural, y reconocer la heterogeneidad de todos los grupos. Así pues, el Instructivo 007 del 2014 ordena explícitamente implementar, en el curso de seis meses desde su fecha de emisión:

a) Normas de participación ciudadana.

b) Creación de una Unidad de Participación Ciudadana en cada repartición.

c) Consejos de la Sociedad Civil.

d) Cuenta Pública Participativa en cada repartición.

e) Registro Nacional de Personas Jurídicas sin Fines de Lucro. 
De manera complementaria, el Programa de Apoyo a la Descentralización Regional (ADR), de la Subsecretaría de Desarrollo Regional y Administrativo, fijó como objetivo fortalecer las capacidades de las instituciones regionales para que sus gobiernos puedan ejercer efectivamente competencias para liderar y articular el desarrollo de sus territorios. Entre estas líneas se destaca el fortalecimiento de las capacidades locales (SUBDERE, 2016).

\section{Consejos de la Sociedad Civil}

La Ley 20500 estableció una institucionalidad que da soporte jurídico a la participación en el país. No obstante, uno de los aspectos más destacados de la nueva normativa fue la creación de los COSOC, concebidos desde una lógica de gobernanza institucionalizada para articular actores relevantes. Si se considera que las experiencias más exitosas de cooperación entre sociedad civil y Estado se ubican en el ámbito local (De la Maza et al., 2012), resulta entendible la expectación que causó el COSOC, el cual reemplazó a los Consejos Económicos y Sociales Comunales (CESCOS) debido a sus problemas y deficiencias, sobre todo, por su baja representatividad (De la Maza \& Flores, 2009). Lo anterior modificó la Ley 18695 Orgánica Constitucional de Municipalidades.

El cosoc posee un carácter consultivo y se implementó en 2014, con el Instructivo Presidencial 007. La normativa lo concibe como una instancia conformada por actores de la sociedad civil de la comuna en que se constituya, por lo cual debe sesionar al menos cuatro veces al año. De él pueden hacer parte organizaciones comunitarias de carácter funcional, personas jurídicas sin fines de lucro, asociaciones y comunidades indígenas, asociaciones gremiales, sindicatos y cualquier actor involucrado con el desarrollo económico, social y cultural de una comuna (Rodríguez \& Uribe, 2013). Ostenta un carácter más autónomo, comparado con el CESCO (Marín \& Mlynarz, 2012), como también consultivo y democrático, siendo su objetivo principal "profundizar y fortalecer la relación entre la sociedad civil organizada y, en este caso, con los municipios, 
en pos de participar en los procesos de consulta asociados a la toma de decisiones y seguimiento de las políticas públicas” (Loyola, 2016, p. 23).

De acuerdo con la ley, los municipios están obligados a conformar sus respectivos COSOC. Sin embargo, lo cierto es que el cumplimiento de esta disposición ha sido precario. Así lo señala el Consejo Para la Transparencia en su trabajo fiscalizador entre los años 2011 y 2015, el cual detectó una muy baja implementación y ausencia de convocatoria en algunos casos. Del mismo modo, se ha cuestionado el real funcionamiento del COSOC en los lugares donde se ha conformado (Loyola, 2016), así como también su verdadera incidencia en la política comunal y la información que de él posee la ciudadanía (Canelo \& Rodríguez, 2013).

Un estudio realizado en 2016 determinó que cuestiones como la voluntad política de los jefes comunales y la educación cívica de quienes conforman el COSOC son factores relevantes para su operatividad, ya que de ellos dependerán no solo las posibilidades de su adecuada conformación, sino también la probabilidad de que a través suyo se puedan exigir derechos y formular propuestas (Loyola, 2016). Por otro lado, se ha constatado una falta de información y representatividad en sus mecanismos de conformación, así como también una baja incidencia en la política comunal (Canelo \& Rodríguez, 2013). Además, se ha señalado que la capacitación de sus integrantes ha sido deficiente, lo que sumado a la falta de interés, ha terminado debilitando su potencialidad (Panelo \& Rodríguez, 2013). Por otro lado, los propios participantes han reconocido problemas en su constitución, referidos principalmente a carencias de educación cívica y procesos legítimos (Olivares, 2015).

La no consideración de estos elementos pone en riesgo al COSOC de convertirse en una instancia de mero cumplimiento normativo, provista de atribuciones más simbólicas que prácticas, o, en su defecto, instrumentales para fines de la administración municipal, tal como ocurrió con el CESCO (Rodríguez \& Uribe, 2013; Herrmann \& Klaveren, 2016). La poca información y una falta de estudios en la materia, hizo imprescindible un abordaje más profundo y pertinente, premisa que 
postula la gobernanza y la deliberación democrática como elementos analíticos claves.

\section{Gobernanza}

Las nuevas formas de organización social, económica y política han planteado desafíos para las condiciones de gobernabilidad de los Estados contemporáneos. Frente a ello, y con el afán de generar vías institucionales más democráticas, legítimas y efectivas para el ejercicio del poder, es que la gobernanza se ha presentado como una alternativa sustentable de gobernabilidad del sistema político (Zurbriggen, 2011).

La gobernanza es un concepto de origen europeo, el cual plantea procesos horizontales de interacción y coordinación, en los que diversos actores — básicamente el Estado, el sector privado y la sociedad civil— toman decisiones conjuntas y estratégicas para problemas comunes, y buscan maneras más efectivas y eficientes de resolverlos (Rodríguez, Lamothe, Barten \& Haggerty, 2010). Tal postulado implica un modo interactivo y multilateral de gobernar (Stoker, 1998), el cual emerge como respuesta a la incapacidad del modelo clásico de gobierno para responder a los problemas de las sociedades contemporáneas (Le Gales, 2009). Así, se apuesta por un trabajo en redes que busca incrementar capacidades y recursos, y por ende, alcances derivados de la asociación sinérgica entre actores (Aguilar, 2014).

Del mismo modo, la gobernanza aborda la cuestión referida a la legitimidad del gobierno, pero no en términos de su institucionalidad. En este caso se apunta más bien a la eficacia directiva de quien gobierna, asumiendo, por tanto, que un desempeño acertado y exitoso no requiere solo de elementos institucionales, sino también políticos y técnicos (Aguilar, 2010).

Si bien la literatura y los actores institucionales coinciden en la sustentabilidad de adoptar la gobernanza como un modo de tratar los problemas públicos, su desempeño práctico se ha visto limitado por diversos factores. Entre ellos destacan la resistencia de la cultura 
política; las dificultades para actualizar los sistemas democráticos; y la falta de interés de los gobiernos por implementar auténticos procesos de participación y empoderamiento de la sociedad civil (Romero \& Farinós, 2011).

Por otra parte, la sola implementación de mecanismos que respondan a una lógica de gobernanza tampoco garantiza una participación efectiva ni el cumplimiento de los objetivos propuestos. Al respecto, destacan tres críticas: el uso instrumental de estas instancias por parte de líderes políticos; la calidad deliberativa asociada a sus actividades; y los problemas relacionados con la representatividad de los participantes (Raya, Espadas, \& Aboussi, 2013). En relación con el último punto, debe reconocerse que el proceso de puesta en práctica de la gobernanza es tan complejo como la composición misma de los actores involucrados ya que sus intereses pueden ser opuestos e ideológicamente contradictorios (Hufty, 2010). En este sentido, la gobernanza es también un proceso que implica relaciones de poder, siendo considerada "un juego de poder, en el cual competencia y cooperación coexisten como reglas posibles” (Whittingham, 2010, p. 222).

Comprender la aplicación de la gobernanza en ámbitos locales requiere, primero, contextualizar los desafíos que esta ha debido enfrentar en Latinoamérica, más aun, considerando su carácter exógeno. De este modo, y en una primera instancia, la gobernanza ha debido cargar con las expectativas asociadas a la modernización de la gestión pública, iniciada en los años noventa, como así también con los requerimientos del sistema de mercado (Jiménez, 2008). En este sentido, destaca la gobernanza local, concepto que releva la respuesta organizada de comunidades locales a las dinámicas globalizadoras de un mercado que los somete a conflictos y cambios que tienen un origen más allá de su autodeterminación. Una gobernanza democrática local puede ser una respuesta potente frente a dicha situación, lo anterior formaría parte del llamado "nuevo localismo" (Bruguè \& Gomà, 1998).

Por otra parte, la constitución histórica de los países de la región también se ha presentado como una variable relevante, destacando 
en ese sentido fenómenos desocializadores y obstaculizadores de los procesos de implementación de gobernanza, tales como el progresivo debilitamiento de la cohesión social, la fragmentación ciudadana y la atomización del mundo laboral (Vitarelli, 2008). Lo anterior adquiere mayor sentido si se repara en su operatividad, la cual recoge el supuesto de una sociedad civil organizada, activa y protagónica, al modo de las sociedades de Europa occidental. Sin embargo, dicho planteamiento es ajeno a la realidad de la mayoría de las sociedades latinoamericanas, en las que la injusticia y exclusión aún afectan a gran parte de la población (Jiménez, 2008).

Buena parte de la responsabilidad de esta falta de adaptación recae en los requerimientos de donantes internacionales como el Banco Mundial (BM) o el Programa de las Naciones Unidas para el Desarrollo (PNUD), quienes han favorecido un modo de implementación del buen gobierno basado en el Estado neoliberal y el funcionamiento del mercado (Zurbriggen, 2011). De forma paralela, la discusión académica e interna del modelo ha sido insuficiente, situación que ha dificultado la puesta en marcha de procesos óptimos que impacten más allá de lo normativo, particularmente por no considerar las condiciones locales de las comunidades (Zurbriggen, 2011), quienes otorgan contenidos ajustados y pertinentes a sus problemáticas. En ese proceso la deliberación democrática juega un rol fundamental.

\section{Deliberación Democrática}

La democracia se considera el gran avance político del siglo xx, bajo el supuesto de que es el modelo político en el que se abren espacios favorables para el mejoramiento de la calidad de vida y la participación de los habitantes de una sociedad (Morlino, 2009). De hecho, se ha afirmado que las sociedades consolidadas democráticamente poseen, en general, mejores perspectivas de desarrollo desde el punto de vista integral de la calidad de vida de las personas (Boutros-Ghali, 2003). Por tanto, se necesitan mecanismos que permitan profundizar y optimizar sus procesos vinculantes. 
Al tiempo que se avanza en la identificación, comprensión y reconocimiento de formas de relaciones de poder que pueden organizarse a partir de redes de interacción, no necesariamente institucionalizadas, también es necesario, por otro lado, analizar la calidad de dichas interacciones, en cuanto a su contenido democrático y respecto a cómo han sido diseñadas, más aún cuando son parte de una política gubernamental (Arellano, Sánchez \& Renata, 2014). Esto último, la calidad del proceso y el diseño, es importante puesto que constituye buena parte del valor que se le asigna a las nuevas formas de organización y distribución del poder en los sistemas políticos en el contexto de la crisis de la democracia representativa.

Si bien los tradicionales sistemas de representación aseguran, en teoría, la legitimidad de un tipo de institucionalidad en las relaciones de poder, también es cierto que desde mediados del siglo XX se viene discutiendo el alcance y calidad de la democracia representativa (Arnstein, 1969; Pateman, 1970). Ello ha requerido comparar dos modelos: uno, en el que las las élites elegidas toman las decisiones y, otro, en el que las bases de la sociedad, los ciudadanos, son quienes las toman de forma directa. Básicamente la cuestión se reduce a si es mejor el gobierno de pocos (elitismo democrático) o el gobierno de muchos (democracia directa) (Weber, 1964; Schumpeter, 1968; Dahl, 1989; Altman, 2010). No obstante, la evidencia demuestra que el par semántico "muchos o pocos" gobernando, no da cuenta de todas las posibilidades analíticas que un ecosistema democrático puede generar o contener, y a que, por supuesto, es necesario avanzar (Reveles, 2017). A partir de lo anterior, el foco de problematización cambia desde las magnitudes en las preferencias en la toma de decisiones hacia la calidad de las relaciones y las formas de definirlas (Martí, 2006).

En este contexto, cobra importancia la deliberación democrática, comprendida como el proceso mediante el cual los sujetos, al interactuar políticamente, llegan a tomar decisiones sobre diversos asuntos a través de mecanismos de discusión, confrontación de ideas, argumentación, búsqueda de consensos y aprendizaje mutuos (Elster, 2001; Cohen, 2007). En este sentido, la idea de la administración del consenso y 
disenso emerge como condición necesaria para arribar a un concepto de calidad democrática vinculado a la deliberación pública.

Si bien la deliberación es un concepto profusamente analizado en teoría política, desde mediados del siglo XX, se ha propuesto su función como componente necesario para aportar un sustento democratizador. Lo anterior, debido a que se considera correctora de las debilidades del sistema de representación y posibilitadora de horizontes de acuerdo a los contextos de conflicto (Renobell, 2017).

Más allá de los planteamientos teóricos que señalan las ventajas de implementar procesos de deliberación, existen componentes empíricos claros capaces de sustentar dicho modelo normativo. Al respecto, es posible identificar cuestiones como el control de las asimetrías de poder; la sustentabilidad material de los procesos deliberativos; la implementación de metodologías de interacción adecuadas; el garantizar la inclusión de todos los actores relevantes, entre otros. No obstante, lo central está dado por la calidad en el desempeño democrático, y por tanto, por las condiciones de participación y los resultados asociados (Gutmann \& Thompson, 2004; Monsiváis, 2008).

Aun así, la perspectiva deliberativa tampoco se encuentra exenta de críticas. Una de ellas apunta a las diferencias que puede haber entre los actores respecto de sus contextos económicos, sociales, políticos y culturales, entendiendo que las inequidades económicas, por ejemplo, no permiten igualdad en sus posiciones e interacciones. Otros puntos dan cuenta de cómo los conflictos políticos impiden planos de consenso, o cómo las culturas o sociedades no poseen condiciones de entrada para procesos de deliberación, al destacar la situación de los pueblos originarios o de las minorías sin reconocimiento de derechos, por ejemplo. Por ello es tan importante resguardar y/o corregir aspectos vinculados a los procesos, tales como la debida operacionalización, la desinformación y las asimetrías de poder, entre otras variables (Bobbio, 2010).

Independientemente de sus deficiencias, lo cierto es que la deliberación se constituye como un componente relevante en los procesos 
que aspiran a constituirse participativa y democráticamente. Respecto de ello, uno de los principales focos de atención debe situarse en el impacto de las decisiones, y, por tanto, en su legitimidad y beneficios. $\mathrm{Al}$ respecto, es importante entender que los procesos deliberativos en la gestión pública no están condicionados por la sola implementación de estructuras participativas, sino por el despliegue de condiciones que permitan a la institucionalidad propiciar y garantizar aspectos como la posibilidad de argumentar, bajo condiciones de inclusión, para desde allí, llegar a acuerdos y tomar decisiones. Todo ello conforma el plano procedimental que se articula en las estructuras de gobernanza disponibles.

\section{Presentación caso de estudio y Metodología}

El caso empírico de estudio es el proyecto titulado "Instalación de Consejo de la Sociedad Civil Regional y Propuesta de dos mecanismos de participación ciudadana de acuerdo a la realidad territorial", ejecutado entre enero y diciembre de 2017, por el Centro de Políticas Públicas y Ciudadanía de la Universidad del Bío-Bío, en la Región del Biobío, Chile. El Gobierno de la Regional del Biobío, en el marco del programa Incide de la Subsecretaría de Desarrollo Regional (SUBDERE), financió este proyecto, cuyo su fin fue realizar las modificaciones tendientes a mejorar los mecanismos de participación, transparencia y control ciudadano regional. ${ }^{2}$ El objetivo del proyecto fue la constitución, regulación y funcionamiento del cosOC Regional para identificar dos mecanismos de participación ciudadana, de acuerdo a la realidad territorial. La metodología consistió tanto en la realización de un diagnóstico mixto (cuantitativo y cualitativo), como en una propuesta de planificación que requirió la ejecución de encuestas, entrevistas y talleres con el fin de recoger datos relativos a las opiniones, percepciones y conocimientos de los actores claves y la ciudadanía en general.

\footnotetext{
2 Para mayor información consultar la web: http://www.descentralizacion.subdere.gov. $\mathrm{cl}$ /pilotajes/participacion.html o http://participacionciudadana.subdere.gov.cl/
} 
En lo concerniente a la presente investigación, definida como mixta, predominantemente cualitativa y provista de alcance descriptivo, este enfoque se justifica y relaciona directamente con la pregunta y el problema que se planteó, ¿de qué forma responde el COSOC a un ejercicio de gobernanza que reúna las condiciones para generar procesos efectivos de deliberación democrática y, por tanto, de óptima participación ciudadana? Es decir, si bien el proyecto base efectuó un trabajo de diagnóstico metodológico mixto simultáneo, nuestras inquietudes de investigación, nos llevaron a esgrimir una pregunta descriptiva que indagara por el funcionamiento y la calidad de la instancia, a la luz de ciertos marcos interpretativos más amplios que lo que el estudio base demandó. En ese sentido, el análisis de la información entregada por los talleres con líderes de instituciones y asociaciones locales constituyó el insumo principal de análisis, presentándose así la información proveniente de las encuestas como complementaria a los resultados cualitativos. Con esto se buscó llevar a cabo un ejercicio de triangulación de métodos capaz de entregar mayor validez a los hallazgos obtenidos (Marradi, Archenti \& Piovani, 2007).

Los datos utilizados han sido extraídos desde la fase de diagnóstico del proyecto, en la cual se buscó conocer el estado de los COSOC en la región del Biobío y se midió la percepción y características de la participación ciudadana. ${ }^{3}$ Ello requirió la aplicación de diversos instrumentos, entre ellos, dos encuestas: una en línea dirigida a los encargados de participación ciudadana, y otra telefónica dirigida a la población en general cuyo objetivo fue evaluar el estado actual de funcionamiento de los cosoc Municipales y de Servicios Públicos. Del mismo modo, se ejecutaron una serie de talleres participativos en las cuatro provincias de la región del Biobío, cuyo objetivo fue identificar problemáticas, diagnóstico y sugerencias para la elaboración de la propuesta de constitución, integración y funcionamiento de los

\footnotetext{
3 Los resultados del diagnóstico realizado se encuentran en los siguientes informes: "diagnóstico y línea base" e "Informe III: Propuesta de instalación consejo de la sociedad civil regional y dos mecanismos de participación ciudadana". Elaborado por la Universidad del Bío-Bío y que tuvo como mandante al Gobierno Regional del Biobío en el marco del proyecto mencionado durante el año 2017.
} 
COSOC. La importancia de los talleres se fundamentó en el componente colectivo asociado a la producción y riqueza de los datos, básicamente porque fue la única instancia en la que los actores sociales e institucionales se encontraron e interactuaron en igualdad de condiciones. Esto último posee especial relevancia en el contexto de un estudio sobre participación ciudadana deliberativa.

\section{Presentación datos descriptivos encuestas encargados de participación y ciudadanía en general}

Hasta septiembre de 2018 la región del Biobío contaba con un total de cincuenta y cuatro comunas distribuidas en cuatro provincias: Arauco, Concepción, Biobío y Nuble. ${ }^{4}$ De acuerdo con el sondeo realizado por el proyecto, específicamente en lo concerniente a la información suministrada por los encargados de participación ciudadana de cada municipio (cincuenta y cuatro encuestas en línea), en 2017, solo un $56 \%$ de las comunas de la región contaban con sus COSOC constituidos, encontrándose el $86 \%$ de ellos en pleno funcionamiento. El número de los integrantes del COSOC depende del lugar donde esté constituido, identificándose un promedio de doce personas. $\mathrm{Al}$ respecto, el número de miembros no es necesariamente proporcional a la población total, destacándose, en ese sentido, las comunas pequeñas como Ranquil (5755 habitantes) con 30 miembros, versus otras mayores como Chillán (184739 habitantes) con 20 integrantes. Otro dato relevante es que la mayor parte de las organizaciones que están presentes en los COSOC son funcionales (adultos mayores, iglesias, entre otras), seguidas de Juntas de Vecinos (JJVV), presentando así un perfil de organizaciones de base y menos de instituciones locales (ONG, corporaciones, entre otras).

Entre las principales razones para justificar la no constitución de los COSOC municipales se argumentó la escasez de organizaciones validadas por el Tribunal Electoral Regional (29\%); la falta de quórum (25\%) y de interés por parte de las organizaciones (25\%); la discontinuidad de

4 Desde el 5 de septiembre 2018 constituida como la nueva región de Nuble. 
las prioridades establecidas por diferentes administraciones (13\%); y el debilitamiento de las organizaciones comunales así como la inexistencia de encargados de participación ciudadana (4\%).

Otro de los datos que arrojó el sondeo indicó que un 93\% de los COSOC municipales declaran conocer la Ley 20500 sobre Participación Ciudadana. Además, la autopercepción referida en cuanto a la utilización de funciones y atribuciones propias del COSOC resultó ser media-baja, ya que solo ocasionalmente se habría hecho uso de las funciones y atribuciones propias del organismo. Al respecto, lo que recibió una valoración mayor fue su capacidad para pronunciarse sobre la eficacia de los servicios públicos con una media de 3,00. ${ }^{5}$ Por el contrario, lo que recibió una menor valoración fue, por una parte, su capacidad para influir en decisiones propias del alcalde — tal como es el llamado a la realización de plebiscitos comunales o la aprobación o modificación del Plan Regulador - y, por otra, la posibilidad de efectuar el levantamiento de recursos de reclamación en contra de resoluciones u omisiones ilegales de la municipalidad, obteniendo ambas una media de 1,14. Dentro de la misma medición, el ítem referido al acceso a recursos municipales necesarios para el funcionamiento del COSOC recibió una alta valoración (la mayor, de hecho), con una media de 4,36.

La encuesta telefónica se realizó a una muestra de 1300 personas entre los 18 y 59 años o más, distribuidas en las cuatro provincias. Dicho instrumento abordó cuatro dimensiones: gestión pública y participación electoral; asociatividad y participación individual; participación ciudadana en la gestión institucionalidad; y marco normativo. Entre sus principales resultados destacan las condiciones de asociatividad y participación presentes en la región y provincias. De este modo, a nivel regional, un 71,8\% de los encuestados declaró haber escuchado hablar

\footnotetext{
5 La escala de valoración fue de 1 a 5, siendo la media de 2,5: valores inferiores representan una baja actitud, valores sobre 2,5 representan una alta actitud. Para identificar la fiabilidad de la información presentada, se sometió la escala de actitud a una prueba estadística, la que es denominada como consistencia interna del instrumento, con el objetivo de conocer si las preguntas que componen el constructo teórico (actitud relativa hacia la utilización de funciones y atribuciones del COSOC del municipio) fueron fiables.
} 
de la participación ciudadana, en tanto solo un 8,3\% dijo conocer la Ley 20 500. Así mismo, un 79,6\% manifestó voluntad de resolver diversos problemas en asociación con vecinos u otras personas. En tanto, un 49,3\% de los encuestados señaló estar muy de acuerdo con la posibilidad de ser parte en alguna instancia institucional de participación. No obstante, un $47 \%$ señaló no saber para qué sirven las instituciones de participación ciudadana.

A continuación, algunos datos relevantes por provincia:

Tabla 1. Disposición total a participar en organizaciones o asociaciones ciudadanas por provincia, región Biobío, 2017

\begin{tabular}{|c|c|c|c|}
\hline Arauco & Biobío & Concepción & Ñuble \\
\hline $44,1 \%$ & $44,3 \%$ & $43,1 \%$ & $48,3 \%$ \\
\hline
\end{tabular}

Fuente: elaboración propia a partir del Informe Gore Biobío, 2017.

Tabla 2. Conocimiento Ley 20500 por provincia, región Biobío, 2017

\begin{tabular}{|c|c|c|c|}
\hline Arauco & Biobío & Concepción & Ñuble \\
\hline $12,7 \%$ & $9,3 \%$ & $3,8 \%$ & $16,1 \%$ \\
\hline
\end{tabular}

Fuente: elaboración propia a partir de Informe Gore Biobío, 2017.

Tabla 3. Porcentaje de personas dispuestas a asociarse para solucionar problemas por provincia, región del Biobío, 2017

\begin{tabular}{|c|c|c|c|}
\hline Arauco & Biobío & Concepción & Ñuble \\
\hline $82,4 \%$ & $87,3 \%$ & $80,1 \%$ & $71,5 \%$ \\
\hline
\end{tabular}

Fuente: elaboración propia a partir de Informe Gore Biobío, 2017.

Tabla 4. Porcentaje de apoyo con afirmación referida a la necesidad de participación de ciudadanos en la confección de leyes y derechos por provincia, región del Biobío, 2017

\begin{tabular}{|c|c|c|c|}
\hline Arauco & Biobío & Concepción & Nuble \\
\hline $82,4 \%$ & $75,9 \%$ & $81,1 \%$ & $72,5 \%$ \\
\hline
\end{tabular}

Fuente: elaboración propia a partir de Informe Gore Biobío, 2017.

Los datos proporcionados por la tabla 1 muestran que la provincia con mayor disposición para participar en asociaciones ciudadanas sería la de Nuble con un 48,3\%. Si bien el conocimiento de la Ley 20500 es muy bajo en todos los territorios, y de acuerdo a la tabla 2, la provincia de Concepción es mínimo y alcanzó allí solo un 3,8\%, 
mientras que en Ñuble este llegó a ser de un 16,1\%. No obstante, respecto a la disposición para solucionar problemas en conjunto con otras personas, y de acuerdo a la tabla 3, el marcador más bajo se localizó en Ñuble con un $71,5 \%$, superado en esta y en las demás provincias por Biobío con un $87,3 \%$. Por último, en la tabla 4 , la vinculación participativa de la ciudadanía en la confección de leyes y políticas obtuvo su mayor apoyo en Arauco con un 75,5\%, mientras que el mínimo se localizó en la provincia de Concepción con un $59,6 \%$.

\section{Talleres participativos}

Los talleres tuvieron como objetivo identificar problemáticas, elaborar un diagnóstico y generar sugerencias para llevar a cabo la propuesta de constitución, integración y funcionamiento del cosoc. La convocatoria incluyó a representantes del cosoc comunal, representantes de funcionarios públicos, organizaciones público-privadas, autoridades e instituciones públicas regionales, empresas e instituciones privadas, representantes municipales y a la sociedad civil.

Se constituyeron talleres por provincia y, aunque no se realizaron en cada comuna, se convocó a personas de todas ellas por un tema de carácter, esencialmente, operativo. El número final y la distribución de los participantes fue la siguiente:

Tabla 5. Participación talleres Proyecto cosoc 2017

\begin{tabular}{|l|l|c|c|}
\hline \multicolumn{1}{|c|}{$\begin{array}{c}\text { Provincias de } \\
\text { Biobío al 2017 }\end{array}$} & \multicolumn{1}{c|}{$\begin{array}{c}\text { Lugar(es) } \\
\text { realización }\end{array}$} & $\begin{array}{c}\text { Cantidad personas } \\
\text { convocadas }\end{array}$ & $\begin{array}{c}\text { Cantidad de } \\
\text { personas asistentes }\end{array}$ \\
\hline $\begin{array}{l}\text { Provincia de } \\
\text { Concepción }\end{array}$ & Concepción & 64 & 47 \\
\hline Provincia de Ñuble & Chillán y Coelemu & 65 & 52 \\
\hline Provincia Biobío & Los Ángeles & 50 & 19 \\
\hline $\begin{array}{l}\text { Provincia de } \\
\text { Arauco }\end{array}$ & Tirúa y Lebu & 62 & 31 \\
\hline
\end{tabular}

Fuente: elaboración propia a partir de Informe Gore Biobío, 2017. 
En el taller se diseñaron cuestionarios individuales que recogieron la opinión de cada participante sobre ciertos tópicos. Posteriormente, se llevó a cabo un ejercicio de discusión grupal en el que se ordenaron y sintetizaron las principales ideas, que fueron plasmadas en una matriz por los facilitadores.

La metodología del taller, desde el punto de vista epistemológico, se ubicó en un paradigma cualitativo que enfatizó en el valor del discurso y de las visiones de los participantes a través del diálogo grupal (Taylor \& Bogdan, 1986). Como técnica específica, los talleres combinaron principios del grupo focal y del análisis de problemas (Ortegón, Pacheco \& Prieto, 2005) y la técnica de taller grupal en lo concerniente al diagnóstico participativo (Geilfus, 2002). El análisis se orientó con la técnica Metaplan (Mosquera, 1999), basada en el supuesto de que a través de la visualización gráfica del discurso, los participantes logran consensuar los contenidos e ideas de modo directo. El diseño se fundamentó en los principios de la participación ciudadana para la gestión deliberativa (Brugué, 2009), es decir, aquellos que resguardan la generación de condiciones de inclusión de actores relevantes, procesos de argumentación, orientación conceptual a la participación y soporte. Todo con el objetivo de fortalecer aprendizajes colectivos y de validar resultados provistos de legitimidad democrática, vinculados a decisiones y conducentes a resultados (León, 2013).

\section{Metodología análisis datos aportados por talleres}

Las unidades de análisis son las respuestas entregadas por los participantes, las cuales se sometieron a un análisis de contenido temático (Baeza, 2002) de tipo deductivo, provisto de cierto número de categorías preestablecidas. El análisis temático transforma los discursos individuales en un metadiscurso colectivo. Las categorías se basaron en algunas de las dimensiones conceptuales que hacían referencia a gobernanza (sentido del discurso, representatividad opiniones, asimetrías de poder) y a deliberación democrática (intercambio de argumentos, resultados vinculados a decisiones). De forma complementaria se 
integraron dos categorías analíticas: los conceptos de capital cultural y de capital social.

Bourdieu define el concepto de capital como "una fuerza inscrita en la objetividad de las cosas que determina que no todo sea igualmente posible e imposible" (2001, p. 132). Por tanto, la distribución de capitales estará determinada por las condiciones inmanentes de la vida social, con toda su complejidad, haciendo del éxito social, por ejemplo, una consecuencia directa del acceso y posesión de diversos tipos de capitales.

Teniendo en cuenta lo anterior, se estimó conveniente y necesario incluir estas categorías a la luz de la discusión teórica y de los requerimientos prácticos de la gobernanza y deliberación. Es decir, de qué manera la participación ciudadana se presenta como una realidad efectiva más allá de los requerimientos normativos de la institucionalidad, cuestión que requiere, en cambio, situar el análisis respecto de las condiciones que presentan los propios actores. Así, el capital cultural, entendido como la instrucción y preparación que poseen los individuos (Bourdieu, 2001), guarda relación con la formación cívica de los ciudadanos y con su consecuente desempeño al interior del espacio de deliberación. El capital social, en tanto, alude al entramado de relaciones interpersonales, a los vínculos sociales y a la representatividad de quienes se supone son la voz de la sociedad civil (Valdivieso \& Davidovics, 2016), y cómo ello repercute en la calidad de la gobernanza.

\section{Principales resultados análisis talleres}

A continuación, se presentan resultados obtenidos del análisis de los discursos producidos en los talleres. Con el objetivo de contextualizar mejor este ejercicio y también de reforzar ciertas ideas, los resultados se acompañan de algunas frases textuales obtenidas en el marco de los talleres. 


\section{Participación y capital social}

El análisis de los talleres indica que las condiciones de capital social varían en cada territorio, presentándose de forma más estable en los actores circunscritos a la Provincia de Concepción. En los demás lugares se encontraría en mayor medida desarticulada o en condiciones de tensión:

[Sobre el cosoc] Hay un objetivo en común, sí, que es el permitir que la comunidad participe, que sea transparente (participante del taller en Concepción).

[Existe una] falta de visión común de los integrantes del COSOC sobre las diferentes problemáticas (participante del taller en Nüuble).

Esta situación es llamativa, no solo porque repercute en la calidad de los procesos de gobernanza, sino porque contradice a los datos cuantitativos previamente presentados por provincia, en donde justamente Concepción es la que presenta los indicadores más débiles de asociatividad. No obstante, se debe considerar que el instrumento en cuestión captó la "disposición" a la asociatividad, más no la situación actual de esta. Independiente de ello, un capital social debilitado afecta los canales de representatividad de los actores, quienes en tanto se encuentren disgregados de sus bases como entre ellos mismos, y son incapaces de transmitir y relevar las necesidades y el sentir de la ciudadanía en los diferentes temas que deben tratarse en el cosoc. La anterior es una situación compleja si consideramos que, justamente, la representación es uno de los tres principales problemas que afectan a la gobernanza (Raya et al., 2013) y que la fragmentación ciudadana, vinculada al fenómeno, no favorece la cohesión social (Vitarelli, 2008) necesaria para la constitución de un espacio que cumpla cabalmente con estas características.

\section{Sentido de los discursos}

La horizontalidad en los discursos y sus relaciones figura como un imperativo dentro de las opiniones de los actores. Eso sí, no como 
una realidad inequívoca, sino más bien como una meta hacia la cual avanzar para favorecer el funcionamiento del cosoc. No obstante, también se reconoce (de manera crítica) la intromisión de modos verticales y dependientes, generalmente contextualizados en la relación del cosoc con los municipios. Esta situación alude a las tradicionales formas de ejercer el poder por parte de los organismos institucionales en Chile (Salazar, 2005), cuya expresión más visible manifiestan los actores de la provincia de Biobío, lugar donde la normalización de las relaciones jerarquizadas ha obstaculizado su funcionamiento: "Incumplimiento de las autoridades en lo pactado... carácter consultivo... autoridad ignora al cosoc" (participante del taller en Biobío). Situaciones como esta revelan una tensión presente entre el proteccionismo de la política representativa (De la maza, 2008) y la intromisión de nuevos espacios de representación ciudadana.

\section{Asimetrías de poder}

Las asimetrías de poder figuran como una constante en las opiniones relativas al funcionamiento del COSOC. Dicho problema se expresa a través de dos situaciones: una es la capacidad de incidencia del cosoC en las decisiones políticas, considerando que se trata de un organismo consultivo. La segunda, en tanto, alude nuevamente a la relación con los municipios, quienes son vistos como una amenaza, potencial o concreta, para la autonomía del espacio en cuestión:

Depende de las voluntades políticas. Una misma política dependerá de la filosofía de la que se aplique (participante del taller en Concepción).

El alcalde de la comuna no debe ser quien tenga autoridad sobre la toma de decisiones (participante del taller en Arauco).

Esto se ajusta con las reservas manifestadas frente al uso instrumental que se le ha dado a la participación ciudadana por parte de instancias políticas (Raya et al., 2013), así como también con el juego de poder vinculado al ejercicio de la gobernanza (Whittingham, 2010). No obstante, y en relación con el planteamiento de Whittingham, 
una cosa es la presencia de diversas pugnas al interior de los espacios de gobernanza, y otra muy distinta es la constante presencia de un actor específico que disputa el control de estas instancias, muy probablemente con un objetivo instrumental. $\mathrm{Al}$ respecto, vale la pena preguntarse por la estructura misma del COSOC y por los posibles resguardos institucionales que deberían tomarse para evitar este cuadro, predecible por demás frente a los antecedentes histórico-políticos del Estado chileno.

\section{Capital Cultural}

La información y preparación de los actores que participan del cosOC es relativamente variable, según la provincia analizada. Sin embargo, un aspecto común a todos ellos es la falta de conocimiento de la Ley 20500 y de la institucionalidad que sostiene esta instancia. Este resultado específico se contrapone a lo informado por los encargados municipales de participación, cuestión que podría indicar que la gran mayoría de los actores, incluidos los municipales, no entenderían muy bien qué es el COSOC, ni cuáles son sus verdaderos objetivos, participando por un requerimiento normativo más que por una conciencia real de incidencia ciudadana en la política pública.

La ciudadanía no tiene información respecto a lo que es COSOC (participante del taller en Arauco).

El problema es la falta de información, se reunían en el COSOC sin saber a qué iban (participante del taller en Concepción).

Por otra parte, no se debe olvidar que uno de los elementos claves de la democracia deliberativa radica en el requerimiento de igualdad, el cual se sustentaría en la calidad de los argumentos esgrimidos durante la discusión (Díaz del Castillo, 2013). En este caso, si los actores no comprenden el objetivo del COSOC ni el valor de la participación ciudadana, ¿es realmente posible esperar la puesta en juego de discusiones provistas de sentido y contenido deliberativo? 


\section{Intercambio de argumentos}

Las condiciones en el intercambio de argumentos están marcadas por las restricciones institucionales del COSOC, la articulación de este con la ciudadanía y la actitud que ella manifiesta frente a dicha instancia. Al respecto, es la falta de un mayor impacto en las decisiones políticas una limitante para la concreción de un trabajo provisto de mayor convocatoria:

Las ideas de los ciudadanos no son tomadas en cuenta, solo se consulta, y eso desanima a participar (participante del taller en Concepción).

Con la realidad del cosoc actual no se promueve la participación (participante del taller en Arauco).

Esta situación, apoyada por el sondeo cuantitativo (incidencia en las decisiones municipales, el ítem con menor puntaje en la encuesta en línea), desincentivaría un intercambio de argumentos más fluido, básicamente porque estos no terminarán afectando de forma significativa las decisiones políticas. Del mismo modo, la falta de conocimiento por parte de la ciudadanía y sus flancos organizativos, sobre todo en lo referido a la participación no electoral, terminarían restringiendo este ejercicio de intercambio, lo cual se vuelve un problema basal para el establecimiento de las relaciones y resoluciones a las que puedan llegar los actores (Martí, 2006).

\section{Resultados vinculados a decisiones}

En sintonía con el punto recién expuesto, resulta esperable la ausencia de resultados significativos vinculados a la gestión del organismo. Sin embargo, es importante especificar que no se trata de una falta de capacidades para la toma de acuerdos o decisiones. Muy por el contrario, los actores manifiestan una alta conciencia respecto del bajo o nulo impacto que posee el COSOC sobre las decisiones políticas, a pesar de no poseer mayor claridad respecto a sus objetivos. Desde esta perspectiva se podría asumir que es la propia condición 
institucional del cosoc la que desalienta un trabajo más enfocado en la toma de decisiones. Paralelamente, resulta peculiar constatar, no obstante lo recién expuesto, cómo la misma limitación institucional de la instancia termina por aunar opiniones, y en función suya, proponer una serie de medidas para cambiar dicha situación. Es decir, es la constitución misma del COSOC el problema que hace posible que los actores lleguen a acuerdos provistos de potenciales resultados:

Que sea resolutivo, incidencia en la toma de decisiones públicas y tener una verdadera representatividad e independencia (participante del taller en Arauco Nuble).

Que los COSOC sean autoconvocados y autogestionados... La mesa directiva del COSOC regional debe ser elegida por los presidentes de los COSOC comunales (54), el presidente de esta mesa elegida obtener las $3 / 4$ de los votos (participante del taller en Arauco Biobío).

En este sentido, se podría decir que la estructura de oferta participativa dada por el Estado es la que no aporta a la calidad de los procesos democráticos (Monsiváis, 2008), cuestión que apunta a las condiciones de operacionalización de la deliberación (Bobbio, 2010). Estas últimas, basadas más en el cumplimiento de normativas que en el cultivo de un apropiado ecosistema de decisiones (Reveles, 2017).

\section{Conclusiones}

Uno de los hallazgos más interesantes consignados en este artículo apunta al protagonismo del cumplimiento normativo de los espacios de participación ciudadana, lógica institucional a través de la cual, hipotéticamente, se garantiza el funcionamiento de órganos como el Cosoc. En los últimos años hemos presenciado la universalización de diversas instancias de asociatividad institucional (consejos, comités ciudadanos, directorios, etc.), que, sin embargo, no siempre consideran las características de los contextos de trabajo, tales como la existencia de demanda de dichos espacios o la instrucción ciuda- 
dana necesaria. Manifestar de forma declarativa una posición favorable para nuevos espacios de incidencia no garantiza condiciones para la gobernanza ni para el ejercicio deliberativo en términos de organización, cohesión, representación e incidencia. Esto a propósito de los dispares porcentajes de personas que se declaran favorables a asociarse o participar vs. quienes realmente han escuchado o tienen conocimiento de la Ley 20500.

Tal dicotomía apunta al conocimiento de derechos y deberes ciudadanos como también a la conciencia del impacto que la participación puede tener en el ámbito de lo público y lo local. A su vez, esto da cuenta de un problema más profundo, relativo al diseño global de la política de participación y cómo esta se hace cargo no solo de los componentes operativos asociados a su implementación, sino también de las variables que repercuten integralmente en la calidad de los procesos. Entre estas se encuentra el débil soporte de los recursos con que cuentan las organizaciones de la sociedad civil en general (Soto \& Viveros, 2016), lo que repercute en limitaciones materiales para el ejercicio de representatividad. Por otro lado, la literatura comparada indica que mecanismos como los consejos son efectivos para la toma de decisiones asociadas a políticas, pero poseen debilidades en cuanto a representación (Font, Blanco, Goma \& Jarque, 2000). En ese sentido, el contexto y sus particularidades territoriales se perfilan como factores fundamentales a considerar en cualquier diseño participativo. No por nada, argumentos como la discontinuidad entre las administraciones municipales o la falta de organizaciones validadas justifican el importante número de COSOC aún no constituidos. En definitiva, los espacios se crean, pero no necesariamente cumplen con su objetivo.

En esta misma lógica, es necesario destacar las debilidades señaladas en los discursos, varias de las cuales se orientan a las capacidades de agenciamiento de los actores. Si bien es cierto que una política puede favorecer el desarrollo de espacios y competencias, también lo es que sus resultados dependan de la capacidad de los sujetos para instalar y tensionar las agendas públicas. Al respecto, insistimos en la importancia del capital social y cultural, ya que de ellos dependerá la adecuada implementación, 
desarrollo y sustentabilidad de los diseños que promuevan espacios de gobernanza y deliberación. El acceso a la información, la educación y la asociatividad emergen aquí como factores claves, necesarios de impulsar y garantizar. La formación para la ciudadanía democrática es clave.

Un punto aparte constituye la relación entre municipio y COSOC. Sin duda la persistencia de lógicas verticales y de los desbalances de poder derivados de un diseño deficiente, impiden la concreción de un espacio de gobernanza local al no cumplir los mínimos requeridos para su desempeño. Al respecto, la cooptación e instrumentalización de este tipo de instancias es un riesgo factible en contextos en los que el cumplimiento aparece como un imperativo que sobrepasa las posibilidades y potencialidades de la participación. Esto, sin duda, tensiona las tradicionales lógicas de gestionar lo local con los nuevos enfoques basados en una gobernanza participativa (Loyola, 2016).

Así, la sobrevaloración del componente normativo de la política y las falencias en el análisis del contexto, junto a las debilidades de los propios actores en términos de formación y asociatividad, se presentan como elementos determinantes en la disparidad y/o limitaciones asociadas a esta experiencia de participación democrática local. En ese sentido, el potencial del COSOC, como espacio de gobernanza democrática local, se ve reducido, al involucrar a un número acotado de actores, quienes no logran configurar redes de incidencia representativa de una comunidad más amplia. Si no se puede codecidir y cogestionar la política, las posibilidades de gobernanza local deliberativa desaparecen (Navarro, 2002).

Desde el punto de vista de la calidad de los procesos de deliberación, es posible identificar varios obstáculos. Entre los principales figuran la concientización del significado de la práctica deliberativa y la necesidad de que estos procesos tengan incidencia en las políticas. El margen para una deliberación de calidad, en contextos de desconfianza y de menor impacto en las decisiones de políticas públicas, desanima a las personas a participar. Paralelamente, es posible que el diseño institucional no sea tan relevante si existe disposición a dialogar por parte de los actores. Es decir, no solo se trata de exigir o demandar 
prácticas de incidencia, sino también de construirlas. Esto es relevante, pues un buen proceso de diálogo e intercambio será un estímulo al aprendizaje, el cual, a su vez, podría impulsar un proceso en el que la democracia "eduque a la democracia". En este sentido, el COSOC, como espacio de gobernanza local, figura más como un proyecto a mediano plazo que un actual espacio de intercambio y de deliberación local. Además, el COSOC municipal se debe concebir en articulación con otros instrumentos complementarios que la propia ley define y con la red de otros consejos con los que forma un entramado más amplio de deliberación y gobernanza.

Por lo anterior, es posible concluir que no se trata solo de crear nuevos organismos o de potenciar experiencias locales democráticas, sino de construir espacios en los que la ciudadanía participe de forma verdaderamente activa y democrática del quehacer público, imperativo que no solo es válido para el caso chileno, sino para cualquier otro contexto en el que se busque fortalecer un sistema de democracia participativa, más aún en un contexto de creciente apatía y desvalorización de la democracia (Corporación Latinobarómetro, 2018). Ello requiere reforzar vínculos asociativos y de formación ciudadana, así como también dimensionar el impacto de la participación en la mejora de la calidad de las políticas.

Con preocupación, constatamos la falta de estrategias para disminuir la brecha entre obligaciones normativas y condiciones de participación que los diversos estudios señalan. En enero de 2017 se entregó el informe titulado "Estado de la participación ciudadana en Chile y propuestas de reforma a la Ley 20500 sobre Asociaciones y Participación Ciudadana en la Gestión Pública", dicho documento fue elaborado por el Consejo Nacional de Participación Ciudadana y Fortalecimiento de la Sociedad Civil, instancia que generó diagnósticos y sugirió medidas concretas a partir de una reformulación de la Ley 20500 y de la profundización de elementos del actual diseño de COSOC municipal. ${ }^{6}$ Nos parece necesario considerar los diversos esfuerzos que

6 Muchas de estas propuestas aun no se han concretado. 
se hacen en investigación para identificar fortalezas y debilidades de estos procesos, los cuales también se constituyen como instancias de aprendizaje y de crecimiento. Así pues, esperamos que en el futuro se puedan generar otros estudios que trasciendan el ámbito regional o particular de los COSOC y que puedan dar pie no solo a conclusiones sobre participación ciudadana sino también a nuevas estrategias para consolidar procesos de gobernanza y de deliberación.

\section{Referencias}

Aguilar, L. F. (2007). El aporte de la Política Pública y de la Nueva Gestión Pública a la gobernanza. Revista del CLAD Reforma y Democracia, (39), 5-32. Aguilar, L. F. (2010). Gobernanza: el nuevo proceso de gobernar. Colecciones Administración Pública. Fundación Friedrich Naumann para la Libertad. Recuperado el 20 de mayo de https://repositorio.uesiglo21.edu.ar/ bitstream/handle/ues21/12820/Gobernanza\%20\%20El\%20nuevo $\% 20$ proceso $\% 20 \mathrm{de} \% 20$ gobernar.pdf? sequence $=1 \&$ is Allowed $=\mathrm{y}$

Aguilar, L. F. (2014). Las dimensiones y los niveles de la gobernanza. Cuadernos de Gobierno y Administración pública, 1(1), 11-36. https://doi. org/10.5209/rev_CGAP.2014.v1.n1.45156

Altman, D. (2010). Democràcia directa, democràcia representativa i apoderament ciutadà. Barcelona: Generalitat de Catalunya, Departament d'Interior, Relacions Institucionals i Participació, Direcció General de Participació Ciutadana.

Arellano, D., Sánchez, J., \& Bartolo, R. (2014). ¿Uno o varios tipos de gobernanza? Más allá de la gobernanza como moda: la prueba del tránsito organizacional. Cuadernos de Gobierno y Administración, 1(2), 117-137. https://doi.org/10.5209/rev_CGAP.2014.v1.n2.47538

Arnstein, S. R. (1969). A Ladder of Citizen Participation. Journal of the American Institute of Planners, 35(4), 216-224. https://doi. org/10.1080/01944366908977225

Baeza, M. (2002) De las metodologías cualitativas en investigación científico-social. Diseño y uso de instrumentos en la producción de sentido. Concepción: Universidad de Concepción.

Bobbio, L. (2010). Types of Deliberation. Journal of Public Deliberation, 6(2), $1-24$. 
Bourdieu, P. (2001). Poder, derecho y clases sociales. Bilbao: Editorial Desclée de Brouwer.

Boutros-Ghali B. (2003). La interacción entre democracia y desarrollo. París: UNESCO.

Brugué, Q., \& Gomà, R. (Coords.). (1998). Gobiernos locales y políticas públicas. Barcelona: Ariel.

Brugué, Q. (2009). Una Administración que habla es una Administración que piensa. En I. Celaya, F. López Martín, S. Castel Gayán, T. Gómez de Valenzuela (Coords.), Participación ciudadana, para una administración deliberativa (pp. 55-72). Aragón: Dirección General de Participación Ciudadana.

Blanco, I., Fleury, S., \& Subirats, J. (2012). Nuevas miradas sobre viejos problemas: Periferias urbanas y transformación social. Gestión y Política Pública, 21(spe), 3-40.

Canelo, P., \& Rodríguez, C. (2013). Funcionamiento para el trabajo de los COSOC. Sistematización encuentro metropolitano de consejeros comunales de la sociedad civil. Instituto de Ciencias Alejandro Lipschutz (ICAL). Recuperado el 13 de junio de 2018 de https: / / kipdf.com/ funcionamiento-y-desafiospara-el-trabajo-de-los-cosoc_5aef52467f8b9a9f1d8b4606.html

Canto, M. (2008). Gobernanza y participación ciudadana en las políticas públicas frente al reto del desarrollo. Revista Política y cultura, (30), 9-37.

Chaguaceda, A., \& Caldera, A. (2016). Democracia en América Latina. Entre el ideal utópico y las realidades políticas. México D. F.: Editorial Fontamara.

Chamorro, M. (2016). Participación ciudadana en América del Sur: institucionalidad y acciones ciudadanas tras la dictadura en Argentina y Brasil. Revista CES Derecho, 7(1), 27-38.

Cohen, J. (2007). Deliberative Democracy. En S. W. Rosenberg (Ed.), Deliberation, Participation and Democracy Can the People Govern? (pp. 2019-236). Nueva York: Macmillan, Palgrave.

Corporación Latinobarómetro (2018). Informe 2018. Recuperado el 20 enero 2019 de http:/ / www.latinobarometro.org/latNewsShowMore. jsp?evYEAR=2018\&evMONTH=-1

Cruz, L. D., \& Mballa, L.V. (2017). Mecanismos de participación ciudadana en las políticas públicas en América Latina. Políticas Públicas, 10(1), 1-25.

Cunill, N. (1991). Participación ciudadana, dilemas y perspectivas para la democratización de los Estados latinoamericanos. Caracas: Centro Latinoamericano de Administración para el Desarrollo. 
Dagnino, E., Olvera, A., \& Panfichi, A. (2006). La disputa por la construcción democrática en América Latina. Veracruz: Fondo de Cultura Económica.

Dahl, R. A. (1989). La poliarquia: participación y oposición. Madrid: Tecnos.

De la Maza, G. (2008). Mecanismos de participación ciudadana en el diseño, implementación y evaluación de políticas públicas. Programa ciudadanía y gestión pública, (Ref: SCT/2008/5). Programa Ciudadanía y Gestión Pública, Universidad de Los Lagos. Recuperado el 25 de junio de 2018 de https:/ / es.scribd.com/document/271061310/DeLa-Maza-Gonzalo-2008-Mecanismos-de-Participacion-Ciudadana-enEl-Diseno-Implementacion-y-Evaluacion-de-Politicas-Publicas

De la Maza, G., \& Flores, D. (2009). Gestión Municipal Participativa, Construyendo Democracia Cotidiana. Santiago de Chile: Universidad de los Lagos.

De la Maza, G. (2011a). Espacio público y participación ciudadana en la gestión pública en Chile: Límites y posibilidades. Polis, 10(30), 1-27. https://doi.org/10.4067/S0718-65682011000300003

De la Maza, G. (2011b). Construcción democrática, participación ciudadana y políticas públicas en Chile (Tesis de doctorado). Universidad de Leiden, Leiden, Países Bajos. Recuperado el 25 de julio de 2018 de http:/ hdl.handle.net/1887/15360

De la Maza, G., Robles, C., Montecinos, E., \& Ochsenius, C. (2012). Redes de política pública y agendas de participación ciudadana en el Chile postransicional, ¿Desafiando la política o recreando sus límites? Gestión y Políticas Públicas, 21(30), 45-86.

Díaz del Castillo, A. (2013). Aproximación al concepto de democracia deliberativa. Revista Ratio Juris, 8(16), 77-104. Recuperado de https:// dialnet.unirioja.es/servlet/articulo?codigo $=4398690$

Elster, J. (2001). La democracia deliberativa. Barcelona: Gedisa.

Escobar, A. (2004). Participación ciudadana y políticas públicas. Una problematización acerca de la relación Estado y sociedad civil en América Latina en la última década. Revista Austral de Ciencias Sociales, (8), 97-108.

Font, J., Blanco, I., Gomà, R., \& Jarqe, M. (2000). Mecanismos de participación ciudadana en la toma de decisiones locales: una visión panorámica. XIV Concurso de Ensayos del CLAD “Administración Pública y Ciudadanía”. Recuperado el 22 abril de 2018 de http:// www.bantaba.ehu.es/formarse/ficheros/view/Joan_Font.pdf? revision $\% 5$ Fid $=79433 \&$ package $\% 5$ Fid $=79311$ 
Garrido-Vergara, L., Valderrama, L., \& Peñafiel, J. (2016). Democracia deliberativa, instituciones y participación ciudadana en América Latina. Revista de Ciencia Politica, 54(2), 255-275. https://www.doi. org/10.5354/0716-1077.2017.44807

Geilfus, F. (2002). 80 herramientas para el desarrollo participativo: diagnóstico, planificación, monitoreo, evaluación. San José: Instituto Interamericano de Cooperación para la Agricultura (IICA).

Gobierno Regional Región del Biobío, GORE Biobío. (2017). Informe Estudio Instalación de Consejos de la Sociedad Civil (COSOC) y propuesta de dos mecanismos de participación ciudadana de acuerdo a la realidad Territorial. Concepción: Universidad del Bío-Bío.

Gutmann, A., \& Thompson, D. (2004). Why Deliberative Democracy. Nueva Jersey: Princeton University Press.

Herrmann, M., \& Klaveren, A. (2016). Disminución de la participación de la población en organizaciones sociales durante los últimos trece años en Chile e implicaciones para la construcción de una política de planificación urbana más participativa. EURE, 42(125), 175-203. https: / / doi.org/10.4067/S0250-71612016000100008

Hufty, M. (2010) Gobernanza en salud pública: hacia un marco analítico. Revista de Salud Pública, 12(1), 39-61.

Jiménez, R. (2008). La Gobernanza, un concepto problemático en América Latina. Foro Latinoamericano de Gobernanza. La Asamblea Constituyente de Venezuela como expresión de especificas tensiones latinoamericanas en el Debate y la práctica de la gobernanza. Foro LatinoAmericano sobre la Gobernanza (FLAG). Recuperado el 24 de mayo de 2018 de http:// www.institut-gouvernance.org/es/analyse/fiche-analyse-327.html

Le Galès, P. (2009). Gobernanza. En L. Boussaguet, S. Jacquot, \& P. Ravinet (Eds.), Diccionario de políticas públicas (260-267). Bogotá: Universidad Externado de Colombia.

León, J. (2013). La participación ciudadana en las Estrategias Regionales de Desarrollo. Estudio de casos de las regiones del Maule y Bío-Bío, Chile (Tesis de doctorado). Universidad Autónoma de Barcelona, Barcelona, España. Recuperado el 23 de abril de 2018 de https://ddd.uab.cat/ pub/tesis/2013/hdl_10803_120552/jla1de1.pdf

León, J. (2014). Análisis de la calidad deliberativa de las Estrategias Regionales de Desarrollo. Estudio de las regiones del Maule y Biobío. Chile. En L. Valderrama \& B. Santander (Coords.), Socializar Conocimientos, 
nro. 2: Observando a Chile desde la distancia (pp. 632-665). Santiago de Chile: RedInche Ediciones.

Levine, D., \& Molina, J. (2007). La calidad de la democracia en América Latina: una visión comparada. América Latina Hoy 45, 17-46.

Loyola, S. (2016). Factores claves en la implementación de los consejos comunales de organizaciones de la sociedad civil en los municipios de Chile (Tesis de maestría). Universidad de Chile, Santiago de Chile, Chile. Recuperado el 10 abril 2018 de http:/ / repositorio.uchile.cl/bitstream/ handle/2250/143063/Factores-claves-en-la-implementaci\%C3\%B3nde-los-consejos-comunales-de-organizaciones-de-la-Sociedad-Civilen-los.pdf?sequence $=1$

Marín, T., \& Mlynarz, D. (2012). Monitoreo a la Normativa de Participación Ciudadana y Transparencia Municipal en Chile. Ciudad Viva, Santiago cómo vamos, Universidad Diego Portales. Recuperado el 5 de mayo de 2018 de http://ciperchile.cl/pdfs/participacion_ciudadana/Monitoreo_Ley_20500.pdf

Marradi, A., Archenti, N., \& Piovani, J. (2007). Metodología de las ciencias sociales. Buenos Aires: Emecé Editores.

Martí, J. L. (2006). La república deliberativa: una teoría de la democracia. Madrid: Editorial Marcial Pons.

Monsiváis, A. (2008). El desempeño deliberativo de la democracia: ¿cómo se observa? En J. Labastida Martín del Campo, M. López \& F. Castanos (Coords.), La democracia en perspectiva: Consideraciones teóricas y análisis de casos (pp. 35-59). México D. F.: Universidad Nacional Autónoma de México (UNAM).

Morlino, L. (2009). Democracia y Democratizaciones. Madrid: Colección Monografías.

Mosquera, C. (1999). Guía Metodológica para facilitadores de desarrollo local sostenible. Democracia y Democratizaciones. Quito: Ediciones Abya-yala.

Navarro, C. (Octubre, 2002). Gobernanza en el ámbito local. Ponencia presentada en el IV Congreso Internacional del CLAD sobre la Reforma del Estado y de la Administración Pública, Lisboa, Portugal.

Olivares, A. (2015). El despelote de los consejos de la sociedad civil. The Clinic. Recuperado el 4 de marzo de 2018 de http://www.theclinic. cl/2015/06/03/el-despelote-de-los-consejos-de-la-sociedad-civil/

O’Donnell, G. (2004). Notas sobre la democracia en América Latina. En F. Esteves (Ed.), La democracia en América Latina. Hacia una democracia 
de ciudadanas y ciudadanos (pp. 11-82). Buenos Aires: Programa de las Naciones Unidas para el Desarrollo (PNUD).

Ortegón, E., Pacheco, J., \& Prieto, A. (2005). Metodología del marco lógico para la planificación, el seguimiento y la evaluación de proyectos y programas. Santiago de Chile: Naciones Unidas, CEPAL.

Panelo, P., \& Rodríguez, C. (2013). Funcionamiento para el trabajo de los COSOC. Sistematización encuentro metropolitano de consejeros comunales de la sociedad civil. Santiago de Chile.

Pateman, C. (1970). Participation and Democratic Theory. Cambridge: Cambridge University Press.

Pressacco, C., \& Rivera, S. (2015). Democracia, participación y espacio local en Chile. Papel Político, 20(1), 65-89. https://doi.org/10.11144/ Javeriana.papo20-1.dpel

Raya, E., Espadas, M., \& Aboussi, M. (2013). Inmigración y ciudadanía activa. Contribuciones sobre gobernanza participativa e inclusión social. Madrid: Ediciones Icaria.

Renobell, V. (2017). Calidad democrática y democracia deliberativa: situación y perspectiva de futuro. En J. J. Marco \& M. Pérez (Coords.), Radiografiando la Democracia, un estudio sobre corrupción, buen gobierno y calidad democrática (258-274). Valencia: Asociación Valenciana de Politólogos y Universidad Cardenal Herrera.

Reveles, F. (2017). Problemas de la representación política y de la participación directa en la democracia. Estudios Políticos, 42, 11-35. https: / / doi.org/10.1016/j.espol.2017.05.007

Rodríguez, C., Lamothe, L., Barten, F., \& Haggerty, J. (2010). Gobernanza y salud: significado y aplicaciones en América Latina. Salud Pública, 12(1), 151-159. https://doi.org/10.1590/S0124-00642010000700011

Rodríguez, C., \& Uribe, A. (2013). Consejos comunales de la sociedad civily participación ciudadana en la gestión municipal. Instituto de Ciencias Alejandro Lipschutz (ICAL). Recuperado el 15 de abril de 2018 de https: / / nanopdf.com/download/consejos-comunales-de-la-sociedad-civil-y-la_pdf

Romero, J., \& Farinós, J. (2011) Redescubriendo la gobernanza más allá del buen gobierno. Democracia como base, desarrollo territorial como resultado. Boletín de la Asociación de Geógrafos Españoles, (56), 295-319.

Salazar, G. (2005). Construcción de Estado en Chile (1800-1837). Democracia de los “pueblos”. Militarismo ciudadano. Golpismo oligárquico. Santiago de Chile: Editorial Sudamericana. 
Sánchez, J. J. (2015). La participación ciudadana como instrumento del gobierno abierto. Espacios Públicos, 18(43), 51-73.

Schumpeter, J. (1968). Capitalismo, Socialismo y Democracia. Madrid: Aguilar.

Soto, F., \& Viveros, F. (2016). Organizaciones de la sociedad civil en Chile: propuestas para financiamiento público y fortalecimiento institucional. Polis Revista Latinoamericana, 15(45), 429-454.

Stoker, G. (1998). Governance as theory: five propositions. UNESCO. Recuperado el 12 de abril de 2018 de http:/ / catedras.fsoc.uba.ar/rusailh/ Unidad $\% 201 /$ Stoker $\% 202002, \% 20$ Governance $\% 20$ as\%20theory, $\% 20$ five $\% 20$ propositions.pdf

Subsecretaría de Desarrollo Regional y Administrativo (SUBDERE). (2016). Guía participación Ciudadana en la Gestión Municipal. Recuperado el 21 de marzo de 2018 de http://participacionciudadana.subdere. gov.cl/sites/default/files/Guia \%20Participacio\%CC\%81n\%20Ciudadana $\% 20$ en $\% 201 \mathrm{l} \% 20 \mathrm{Gestion} \% 20 \mathrm{Municipal}(4)$.pdf

Taylor, S. J., \& Bogdan, R. (1986). Introducción a los métodos cualitativos de investigación. México D. F.: Paidós.

The Economist. (2017). Democracy Index 2017: Free speech Under Attack. A report by The Economist Intelligence Unit. Recuperado el 12 de julio de 2018 de https://cisp.cachefly.net/assets/articles/attachments/72810_democracy_index_2017.pdf

Valdivieso, P., \& Davidovics, G. (2016). Enfoques y metodologias para la investigación empírica del capital social. Santiago de Chile: RIL Editorial.

Vitarelli, M. (2008). Gobernanza, ciudadanía y democracia. El desafío latinoamericano. Entelequia revista interdisciplinar, (8), 29-39.

Weber, M. (1964). Economía y sociedad: esbozo de sociología comprensiva. México D. F.: Fondo de Cultura Económica.

Welp, Y. (2016). La participación ciudadana como compromiso democrático. Revista Mexicana de Derecho Electoral, (10), 97-121. https://doi. org/10.22201/iii.24487910e.2016.10.11122

Whittingham, M. (2010). Qué es la gobernanza y para qué sirve. Revista Análisis Internacional, (2), 219-235.

Zurbriggen, C. (2011). Gobernanza: una mirada desde América Latina. Perfiles Latinoamericanos, (38), 39-64. 